\title{
Discrete Multiloop, Modified Multiloop, and Plate-Loop Antennas-Multifrequency and Wide-Band VSWR Characteristics
}

\author{
Hisamatsu Nakano, Fellow, IEEE, Mitsutoshi Fukasawa, and Junji Yamauchi, Member, IEEE
}

\begin{abstract}
Three types of loop antennas are presented: discrete multiloop (ML), modified ML, and plate-loop (PL) antennas. The discrete ML and modified ML antennas are composed of $N$ square loops. The $N$ square loops of the modified ML antenna are connected by wires at the loop corners. The analysis of the discrete ML antenna shows that one of the $N$ loops resonates when its circumference is approximately one wavelength. It follows that the discrete ML antenna has $N$ minima in the frequency response curve of the VSWR. In contrast to the discrete ML antenna, the modified ML has a VSWR with a wide-band frequency response: approximately $16 \%$ with $N=7$, which is more than 2.5 times as wide as that for a single-loop antenna $(N=1)$. Further analysis reveals that the PL antenna has a VSWR bandwidth similar to that of the modified ML antenna. The maximum gain of the PL antenna is approximately $9 \mathrm{~dB}$, which is very close to those of the discrete and modified ML antennas.
\end{abstract}

Index Terms-Broad-band antennas, loop antennas, multifrequency antennas, wire antennas.

\section{INTRODUCTION}

I $\mathrm{N}$ MOST applications, the loop antenna is composed of a single round- or square-shaped wire [1], [2]. Although some investigations have been performed for antennas where the number of loops located in the same plane is more than two [3], a detailed investigation of such multiloop (ML) antennas is required to aid in their design. For this requirement, this paper presents three types of loop antennas and reveals their theoretical radiation characteristics. Special attention is paid to the VSWR's of these antennas.

First, an antenna of composed of $N$ loops, which are discrete, is investigated. This kind of antenna is designated as a discrete ML antenna. The discrete ML antenna is excited using proximity coupling from an $L$-shaped wire (simply called an " $L$ wire") [4]-[8]. Note that the feed energy is transferred to the discrete ML antenna by the $L$ wire without any physical contact. The antenna characteristics, including the input impedance, radiation pattern, and gain, are evaluated using the method of moments [9], [10]. Multifrequency (multiresonant) operation is revealed as a function of the number of loops, $N$.

Second, the investigation is directed toward a modified ML antenna in which the corners of the $N$ loops are connected by wires. The method of moments is again used for analyzing the

Manuscript received May 16, 2001.

The authors are with the College of Engineering, Hosei University, Koganei, Tokyo 184-8584, Japan (e-mail: nakano@k.hosei.ac.jp).

Publisher Item Identifier S 0018-926X(02)02612-1.

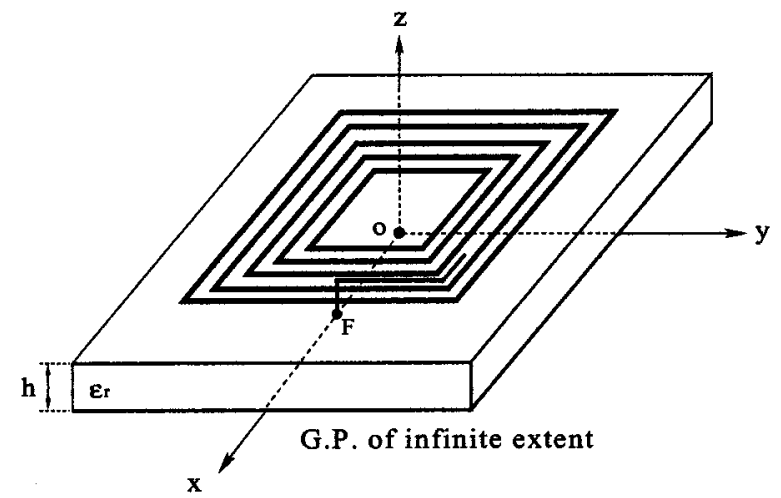

(a)

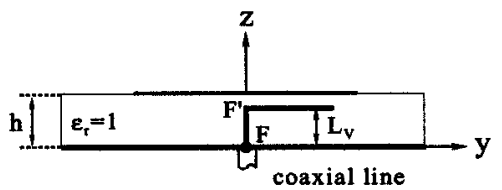

(b)

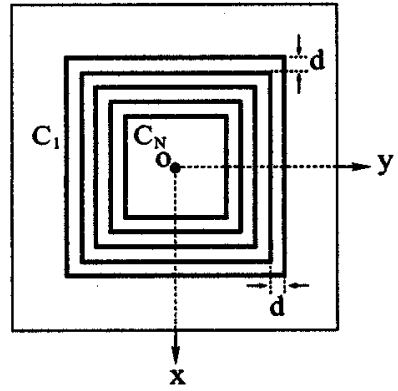

(c)

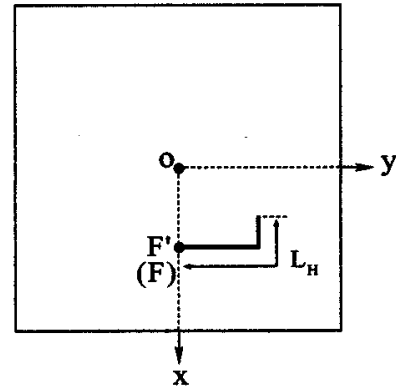

(d)
Fig. 1. Configuration and coordinate system of a discrete multiloop antenna having a ground plane of infinite extent. (a) Perspective view. (b) Cross-sectional view. (c) Top view of $N$ loops. (d) Top view of $L$-shaped wire.

modified ML antenna. The VSWR, radiation pattern, and gain are discussed. It is revealed that the modified ML antenna has a wide-band VSWR frequency response, unlike the discrete ML antenna.

Finally, a plate-loop (PL) antenna is presented. The $L$ wire is used for feeding the PL, as in the discrete and modified ML antennas. The PL antenna is regarded as a modified ML antenna with infinite loops $(N=\infty)$. The analysis of the PL antenna is performed using the finite-difference time domain (FDTD) method [11]-[13]. It is found that the VSWR frequency response is close to that of the modified ML antenna. 


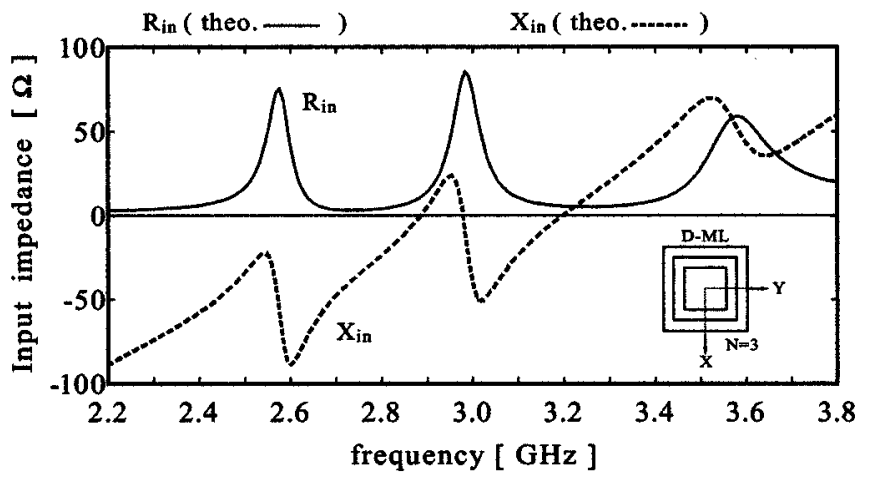

(a)

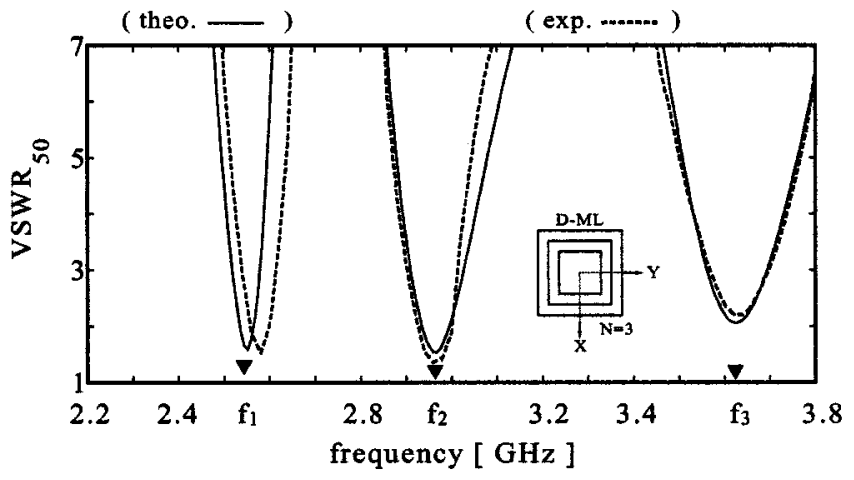

(b)

Fig. 2. Frequency response of a discrete multiloop antenna with $N=3$. (a) Input impedance. (b) VSWR relative to a $50 \Omega$ line.

\section{Discrete Multi-Loop (ML) Antennas}

\section{A. Configuration and Numerical Method}

Fig. 1 shows the configuration of a discrete ML antenna. $N$ discrete loops are located at the same height $h$. These discrete loops, supported by a spacer of relative permittivity $\varepsilon_{r}$, are backed by a conducting plane of infinite extent. The $i$ th loop ( $i=1,2 \ldots N$, counted from the outermost loop) has a square shape of side length $S_{i}$ (circumference of $C_{i}=4 S_{i}$ ). In this paper, the number of loops, $N$, is chosen to be odd, and hence the middle loop is the $(N+1) / 2$ th loop. The discrete ML antenna is excited using an $L$ wire along the middle loop. The $L$ wire, whose vertical and horizontal line lengths are, respectively, $L_{V}$ and $L_{H}$, is connected to the inner conductor of a coaxial line at point $F$ (feed point). The feed point $F$ is on the $x$-axis: $F(x, y, z)=F\left(\left(C_{N}+C_{1}\right) / 16,0,0\right)$. Note that the $L$ wire is coupled to the loops without any physical contact (proximity coupling) [4]-[8].

Based on the circumference of a conventional single-loop antenna for axial beam radiation [1], the outermost and innermost loop circumferences of the discrete ML antenna, $C_{1}$ and $C_{N}$, are chosen to be slightly larger and smaller than one wavelength at a test frequency, respectively. In this paper, these circumferences are arbitrarily chosen to be $C_{1}=1.19 \lambda_{3}$ and $C_{N}=0.870 \lambda_{3}$ (these are also used for modified ML antennas in Section III), where $\lambda_{3}$ is the free-space wavelength at a test frequency of 3 $\mathrm{GHz}$. Note that the relative permittivity $\varepsilon_{r}$ and antenna height

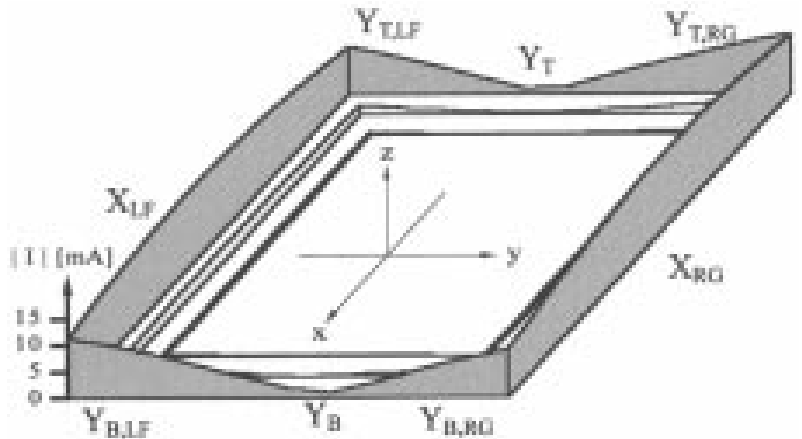

(a)

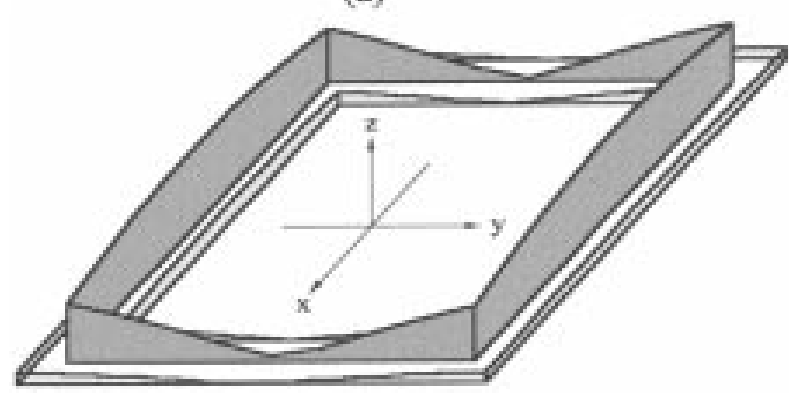

(b)

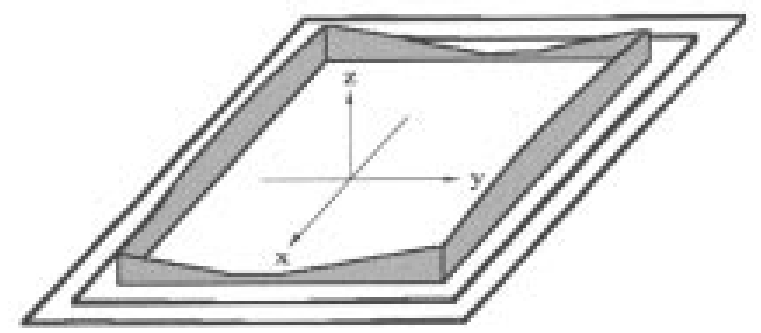

(c)

Fig. 3. Current-amplitude distributions of a discrete multiloop antenna, $|I|$, with $N=3$. (a) At $f_{1}=2.552 \mathrm{GHz}$. (b) At $f_{2}=2.980 \mathrm{GHz}$. (c) At $f_{3}=$ $3.624 \mathrm{GHz}$.

$h$ are fixed throughout this paper: $\varepsilon_{r} \approx 1$ (honeycomb spacer) and $h=0.1 \lambda_{3}$.

The distance between the outermost and innermost loops is $\left(C_{N}-C_{1}\right) / 8=4 \mathrm{~mm}=0.04 \lambda_{3}$, and the middle loop circumference is $C_{(1+N) / 2}=\left(C_{N}+C_{1}\right) / 2=1.03 \lambda_{3}$. The loop-wire radius $\rho_{\text {loop }}$ and $L$ wire radius $\rho_{L}$ are chosen to be the same: $\rho_{\text {loop }}=\rho_{L}=0.002 \lambda_{3}$ (these values are also used for modified ML antennas in Section III). The number of loops, $N$, loop circumferences $C_{i}$ except for $i=1$ and $N$, and center-to-center distance between adjacent wires, $d$, are varied subject to the objectives of the analysis. These are a function of $N$

$$
\begin{aligned}
C_{i} & =\left[(N-i) C_{1}+(i-1) C_{N}\right] /(N-1) \\
d & =\left(C_{1}-C_{N}\right) / 8(N-1)
\end{aligned}
$$

Analysis of the ML antenna is performed using an electric field integral equation [9]. Applying the Method of Moments (MoM) [10] to the integral equation, the current along the wire is numerically obtained. The antenna characteristics are evaluated on the basis of the obtained current distribution. 

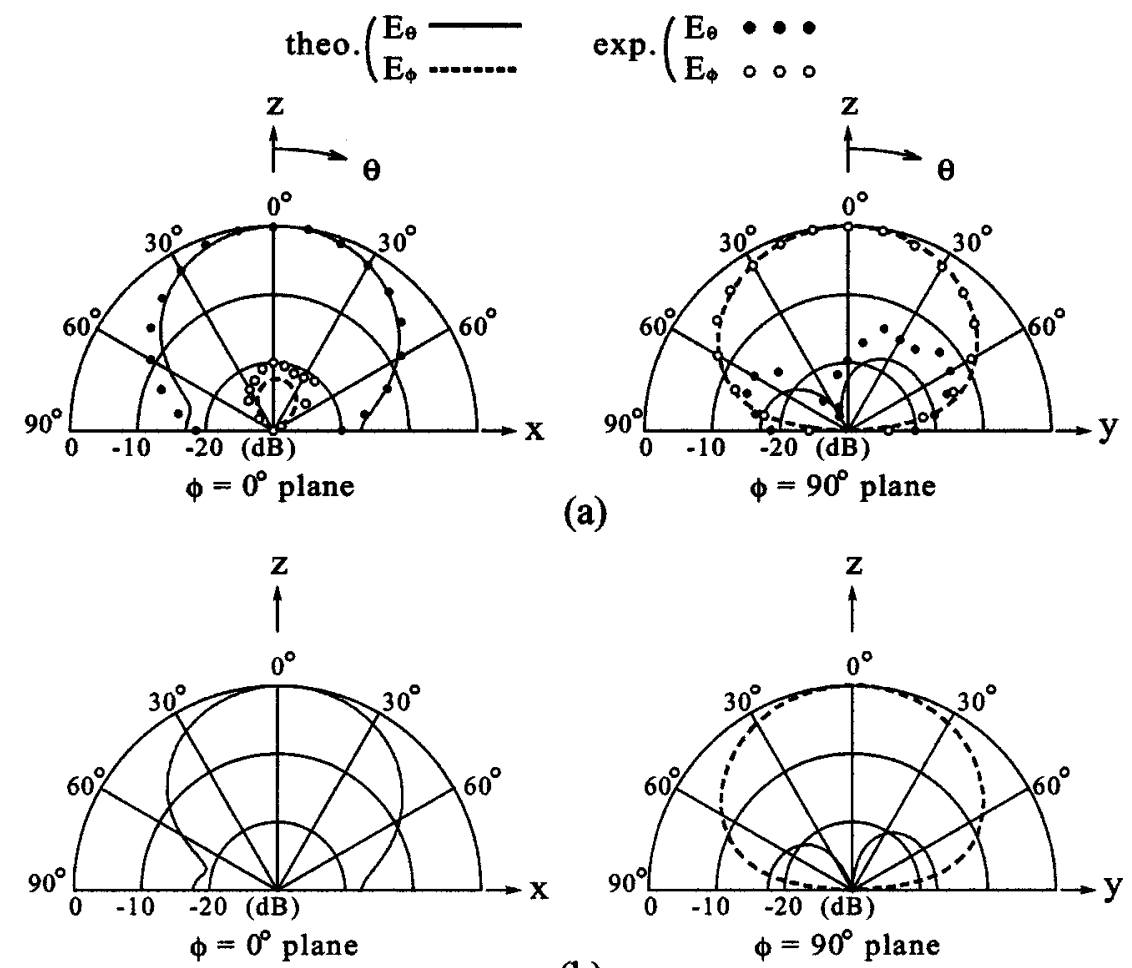

(a)
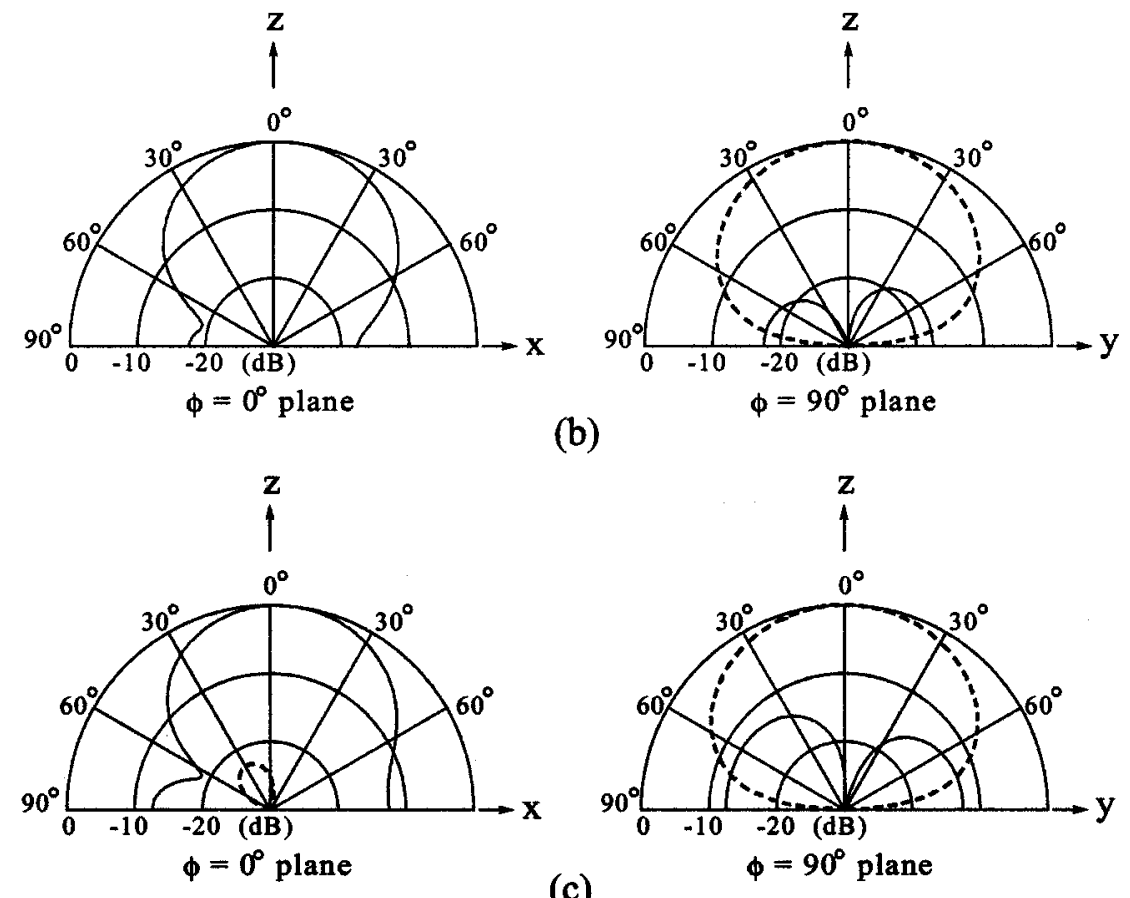

(b)

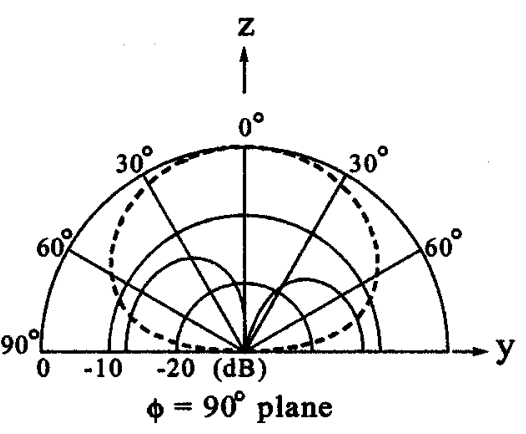

(c)

Fig. 4. Radiation patterns of a discrete multiloop antenna with $N=3$. (a) At $f_{1}=2.552 \mathrm{GHz}$. (b) At $f_{2}=2.980 \mathrm{GHz}$. (c) At $f_{3}=3.624 \mathrm{GHz}$.

\section{B. Discrete 3-Loop Antenna}

First, the number of loops is chosen to be $N=3$. From (1) and (2), the loop circumferences $C_{i}$ and adjacent wire distance $d$ are given as $\left(C_{1}, C_{2}, C_{3}, d\right)=$ $\left(1.19 \lambda_{3}, 1.03 \lambda_{3}, 0.870 \lambda_{3}, 0.02 \lambda_{3}\right)$. The length of the $L$ wire is chosen to be $L_{V}+L_{H}=0.051 \lambda_{3}+0.199 \lambda_{3}=\lambda_{3} / 4$. Figs. 2(a) and (b) show, respectively, the input impedance $Z_{\text {in }}\left(=R_{\text {in }}+j X_{\text {in }}\right)$ and VSWR relative to $50 \Omega\left(\right.$ VSWR $\left._{50}\right)$ as a function of frequency $f$. To verify the validity of the theoretical results, experimental results (dashed lines) are also presented in Fig. 2(b). For this experiment, a large conducting plane of $5 \lambda_{3} \times 5 \lambda_{3}$ is used to approximate the theoretical ground plane of infinite extent. Note that the same conducting plane is used for the following experiment work.

As seen from Fig. 2(b), three minima of the $\mathrm{VSWR}_{50}$ are obtained at three frequencies $f_{i}(i=1,2,3): f_{1}=2.552$ $\mathrm{GHz}, f_{2}=2.980 \mathrm{GHz}$, and $f_{3}=3.624 \mathrm{GHz}$. This reveals that, at each $f_{i}$, one of the three loops has an electrical length of approximately one wavelength: $C_{1}=1.012 \lambda_{2.552}$ at $f_{1}, C_{2}=$
$1.023 \lambda_{2.980}$ at $f_{2}$, and $C_{3}=1.051 \lambda_{3.624}$ at $f_{3}$, where $\lambda_{f}$ is the wavelength at frequency $f \mathrm{GHz}$.

Fig. 3 shows the amplitude distributions of the currents, $|I|$ (current $I=I_{r}+j I_{i} ;|I|=\left(I_{r}^{2}+I_{i}^{2}\right)^{1 / 2}$ ), along the three loops at each frequency $f_{i}(i=1,2,3)$ mentioned above. The largest current flows along the loop whose electrical length is approximately one wavelength at that frequency. That is, one of the three loops resonates at $f_{i}$. Detailed calculations reveal the fact that the currents along the left and right $x$-directed wires of the resonant loop (for example, the currents $X_{\mathrm{LF}}$ and $X_{\mathrm{RG}}$ in Fig. 3(a)) have an in-phase relationship. In addition, the calculations show that the currents along the $y$-directed top wire (for example, $Y_{T, \mathrm{LF}}$ and $Y_{T, \mathrm{RG}}$ in Fig. 3(a)) have an opposite-phase relationship with respect to the amplitude minimum point $Y_{T}$. The currents along the $y$-directed bottom wire [for example, $Y_{B, \mathrm{LF}}$ and $Y_{B, \mathrm{RG}}$ in Fig. 3(a) ] also have an opposite-phase relationship with respect to the amplitude minimum point $Y_{B}$. Due to these phase relationships, the maximum radiation occurs in the $z$-direction, with $x$-directed polarization, as shown in Fig. 4 , which are obtained at the above $f_{i}(i=1,2,3)$. 
The radiation field in Fig. 4 is decomposed into $E_{\theta}$ and $E_{\phi}$ components using the spherical coordinates $(R, \theta, \phi)$. It is found that the radiation patterns for the copolarization components ( $E_{\theta}$ in the $\phi=0^{\circ}$ plane and $E_{\phi}$ in the $\phi=90^{\circ}$ plane) do not significantly change at $f_{i}(i=1,2,3)$. This is due to the fact that the largest current, which obviously makes the greatest contribution to the radiation pattern, always flows along a resonant loop (the loop with an electrical length of approximately one wavelength at the corresponding frequency). Further calculations show that the gain $G$ is approximately $9 \mathrm{~dB}$ at each resonant frequency $f_{i}: G=9.01 \mathrm{~dB}$ at $f_{1}, 9.02 \mathrm{~dB}$ at $f_{2}$, and $8.60 \mathrm{~dB}$ at $f_{3}$.

\section{Discrete 5- and 7-Loop Antennas}

In this section, discrete 5- and 7-loop antennas are analyzed to obtain five and seven minima in the VSWR frequency response, respectively. Note that the $L$ wire for the feed has the same values used for $N=3: L_{V}=0.051 \lambda_{3}$ and $L_{H}=0.199 \lambda_{3}$.

Fig. 5(a) shows quintuple-frequency operation of the discrete 5-loop antenna. The antenna has five $\mathrm{VSWR}_{50}$ minima at five frequencies $f_{i}(i=1,2, \ldots, 5)$. As in the discrete 3-loop antenna, at a given $f_{i}$, one of the five loops has an electrical length of approximately one wavelength. The theoretical and experimental results are in good agreement. The antenna shows a gain of approximately $G=9 \mathrm{~dB}$ at each $f_{i}:\left(C_{1}, G\right)=\left(1.012 \lambda_{2.55}, 8.99 \mathrm{~dB}\right)$ at $f_{1}=2.55$ $\mathrm{GHz},\left(C_{2}, G\right)=\left(1.009 \lambda_{2.728}, 8.76 \mathrm{~dB}\right)$ at $f_{2}=2.728 \mathrm{GHz}$, $\left(C_{3}, G\right)=\left(1.014 \lambda_{2.952}, 8.80 \mathrm{~dB}\right)$ at $f_{3}=2.952 \mathrm{GHz}$, $\left(C_{4}, G\right)=\left(1.023 \lambda_{3.232}, 9.02 \mathrm{~dB}\right)$ at $f_{4}=3.232 \mathrm{GHz}$, and $\left(C_{5}, G\right)=\left(1.051 \lambda_{3.624}, 8.60 \mathrm{~dB}\right)$ at $f_{5}=3.624 \mathrm{GHz}$.

Multifrequency operation is also found in a discrete 7-loop antenna, as shown in Fig. 5(b). The gain for the discrete 7-loop is approximately $9 \mathrm{~dB}$, as is the case for the discrete 3-loop and 5-loop antennas. In other words, the number of loops does not significantly change the gain at each resonant frequency.

\section{Modified Multiloop AnTEnNas}

In Section II, it is found that discrete ML antennas have multifrequency operation corresponding to the number of loops $N$, showing an almost constant gain. In this section, a modified ML antenna shown in Fig. 6 is analyzed. The modified ML antenna has the same structure shown in Fig. 1 except that the corners of the $N$ loops are connected by wires. In the following analysis, the radii of these connection wires are taken to be the same as those of the loops (i.e., $\rho_{\text {loop }}=0.002 \lambda_{3}$ ). The configuration parameters, including $C_{1}, C_{N}, \rho_{L}, h$, and $\varepsilon_{r}$, and the coordinates of the feed point $F$ are defined in Section II.A. The loop circumferences $C_{i}(i=2,3, \ldots N-1)$ and adjacent wire distance $d$ are again defined by (1) and (2).

The solid line $M$-ML in Fig. 7(a) shows the theoretical frequency response of the $\operatorname{VSWR}_{50}$ for $N=3$, together with the experimental result (dashed line), where the $L$ wire has a total length of approximately one quarter-wavelength at $3 \mathrm{GHz}$ : $L_{V}+L_{H}=0.057 \lambda_{3}+0.204 \lambda_{3}=0.261 \lambda_{3}$. It is found that the frequency response of the $\mathrm{VSWR}_{50}$ is smooth, unlike those shown in Figs. 2 and 5. This smoothness results from the

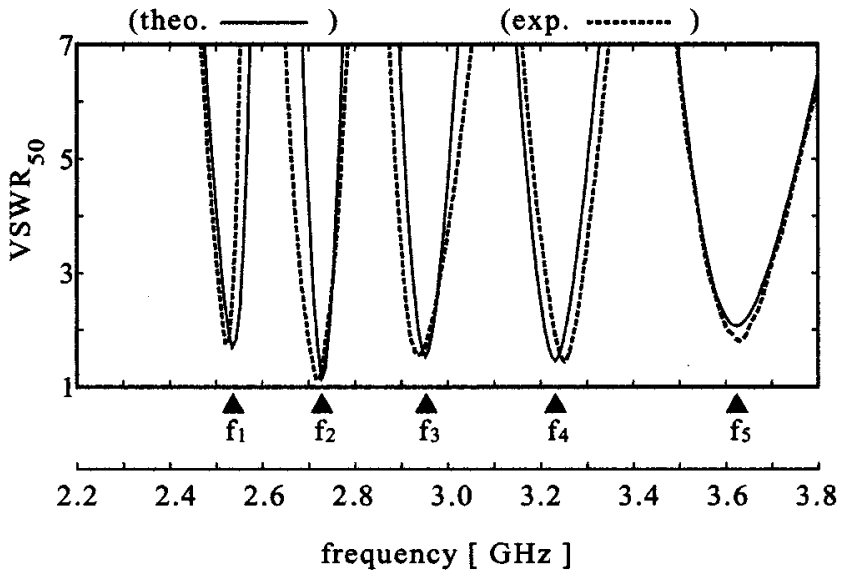

(a)

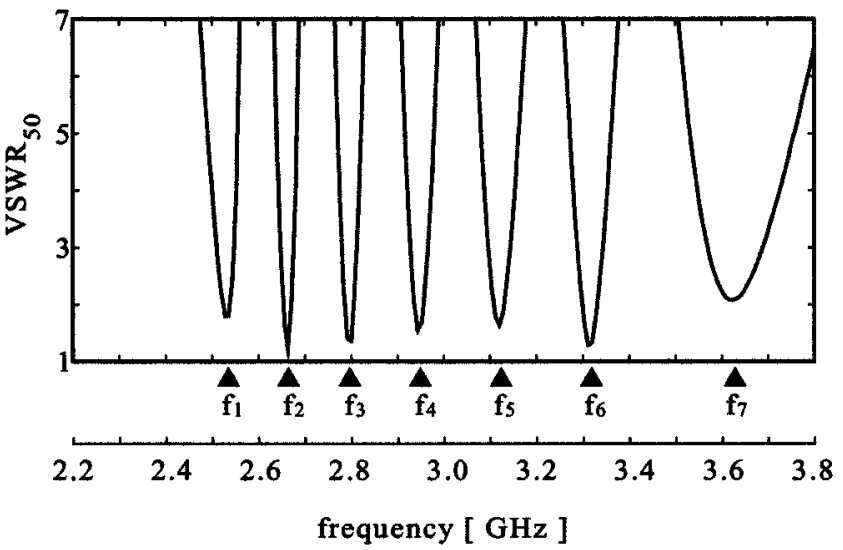

(b)

Fig. 5. VSWR relative to a $50 \Omega$ line. (a) A discrete multiloop antenna with $N=5$. (b) A discrete multiloop antenna with $N=7$.

connection wires at the four corners of the loops. The connection wires make the current distribute along all three loops (see Fig. 8), which differs from the current distribution along the discrete ML antenna, Fig. 3(a). The bandwidth for a $\operatorname{VSWR}_{50}=2$ criterion is $14.9 \%$. Further investigation reveals that the VSWR bandwidth is $15.8 \%$ for $N=5$ and $15.9 \%$ for $N=7$ with an $L$ wire of the same length $0.261 \lambda_{3}$ for each case. These bandwidths are more than 2.5 times as wide as the bandwidth of a single-loop antenna (5.7\%). Note that the VSWR $\mathrm{VS}_{50}$ for the single loop is presented by the "single-loop" line in Fig. 7(a), where the loop circumference is the same as that of the center loop for the discrete ML antennas in Section II: $C=1.03 \lambda_{3}$.

If further reduction of the VSWR is required, the lengths of the $L$ wire components, $L_{V}$ and $L_{H}$, must be changed. Fig. 7(b) shows an example for $N=3$, where $L_{V}+L_{H}=0.045 \lambda_{3}+$ $0.220 \lambda_{3}=0.265 \lambda_{3}$, which is slightly longer than that of the above case $\left(L_{V}+L_{H}=0.057 \lambda_{3}+0.204 \lambda_{3}=0.261 \lambda_{3}\right)$. The frequency bandwidth for a $\operatorname{VSWR}_{50}=1.5$ criterion is calculated to be $5.5 \%$.

The gain and radiation pattern are also analyzed for the $L$ wire having a length of $0.261 \lambda_{3}$, which yields the VSWR curves in Fig. 7(a). The analysis reveals that the gains for $N=3,5$, and 7 as a function of frequency show similar behavior. The maximum 


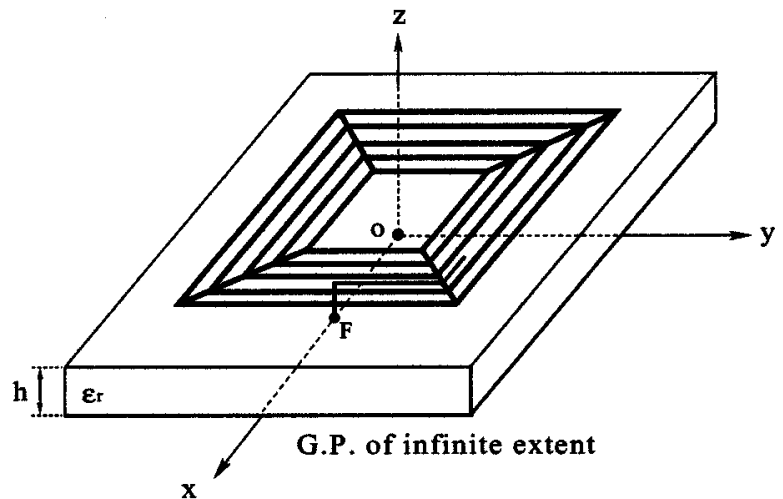

(a)

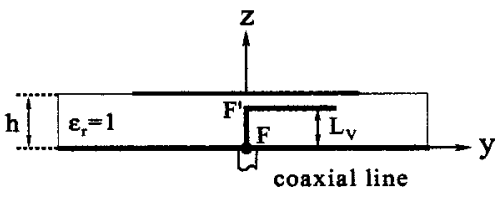

(b)

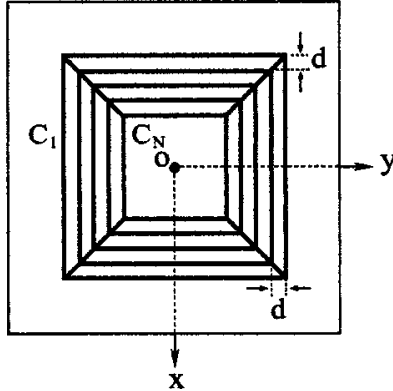

(c) (d)

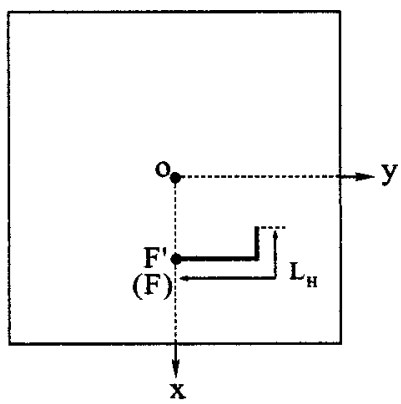

Fig. 6. Configuration and coordinate system of a modified multiloop antenna having a ground plane of infinite extent. (a) Perspective view. (b) Cross sectional view. (c) Top view of $N$ loops. (d) Top view of $L$-shaped wire.

gain is approximately $9 \mathrm{~dB}$, as in the discrete ML antennas. The gain drop from the maximum gain is small (approximately $1 \mathrm{~dB}$ ) within the frequency bandwidth for a $\mathrm{VSWR}_{50}=2$ criterion. Fig. 9 shows the radiation patterns for $N=3$ at $3 \mathrm{GHz}$. Note that the radiation patterns for $N=5$ and 7 are similar to those in Fig. 9.

\section{Plate-Loop (PL) Antenna}

So far, multifrequency operation of a discrete ML antenna and wide-band VSWR characteristic of a modified ML antenna have been discussed. In this section, a PL antenna, shown in Fig. 10, is analyzed. The PL antenna corresponds to the structure when the number of loops, $N$, is infinite in the modified ML antenna case, or to a microstrip patch antenna with a square hole in the patch. The PL antenna is excited using an $L$ wire as the discrete and modified ML antennas. For analysis, the finite-difference time domain method [11] is adopted. The antenna analysis space is subdivided into many Yee cells [12], each having side lengths $\Delta_{x}, \Delta_{y}$, and $\Delta_{z}$, and truncated with Liao's second-order absorbing boundary condition [13]. The cell size in this paper is chosen to be $\Delta_{x}=\Delta_{y}=\Delta_{z}=0.0065 \lambda_{3}$.

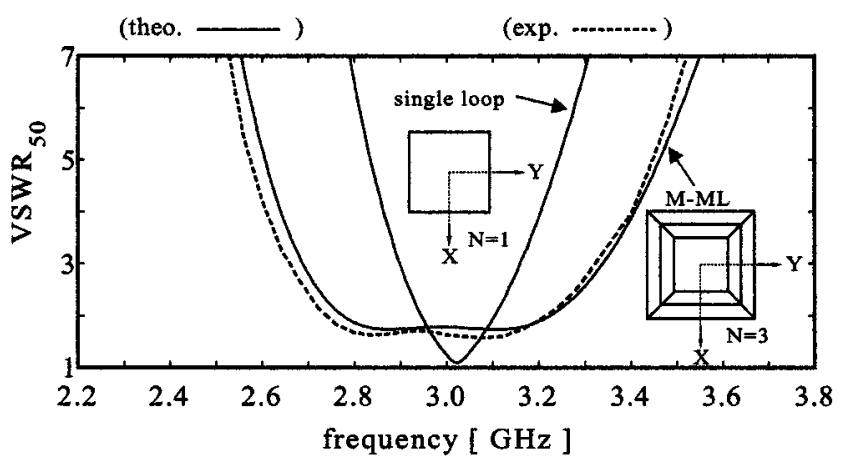

(a)

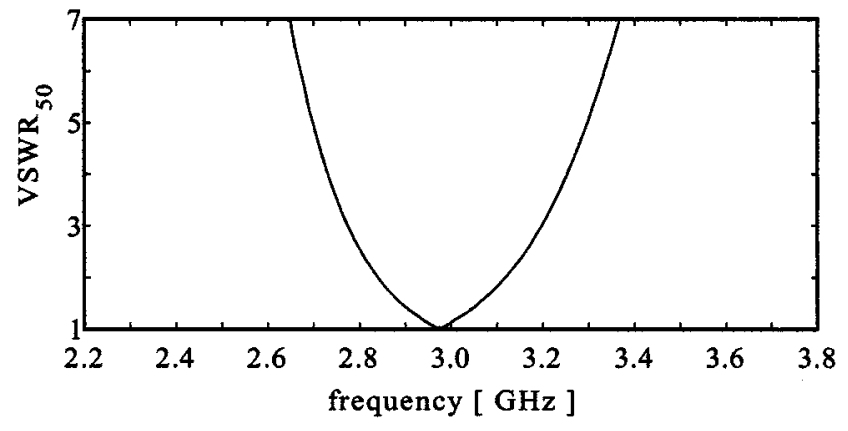

(b)

Fig. 7. VSWR (relative to a $50 \Omega$ line) of a modified multiloop antenna with $N=3$. (a) Together with $N=1$. (b) Lower VSWR 50 .

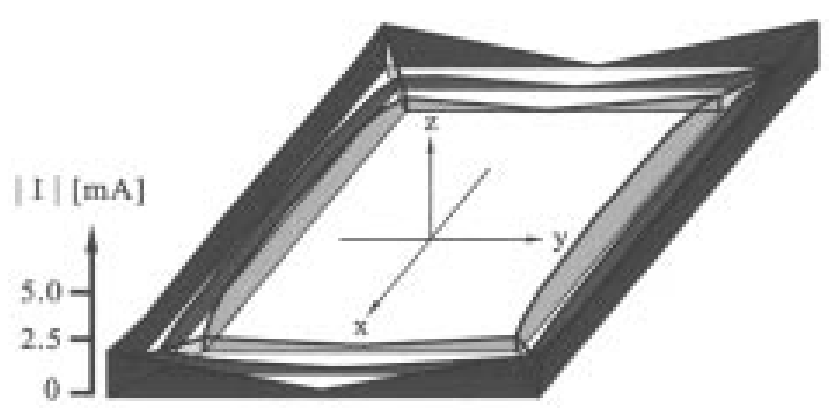

Fig. 8. Current distribution of a modified multiloop antenna with $N=3$ at 3 $\mathrm{GHz}$

The electric field $\mathbf{e}$ and magnetic field $\mathbf{h}$ obtained in the time-domain are Fourier-transformed to $\mathbf{E}$ and $\mathbf{H}$ in the frequency domain, respectively. The antenna characteristics are calculated on the basis of these $\mathbf{E}$ and $\mathbf{H}$. For example, the radiation field is calculated by using the equivalence principle [14], where the electric current density $\mathbf{J}_{s}(=\mathbf{n} \times \mathbf{H})$ and magnetic current density $\mathbf{M}_{s}(=\mathbf{E} \times \mathbf{n})$ are integrated over an equivalence surface, where $\mathbf{n}$ is the outward unit vector normal to the equivalence surface. The current that is necessary for the input impedance calculation is obtained by integrating the magnetic field $\mathbf{H}$ around the $L$ wire at the feed point. The gain $\mathbf{G}$ is calculated using the radiation field, input impedance, and current at the feed point.

The parameters $C_{1}$ and $C_{N}$ defined in Section II.A are used for the width of the PL: $W=\left(C_{1}-C_{N}\right) / 8=0.04 \lambda_{3}$. Fig. 11 shows the components of the electric current density $\mathbf{J}_{s}$ (= 


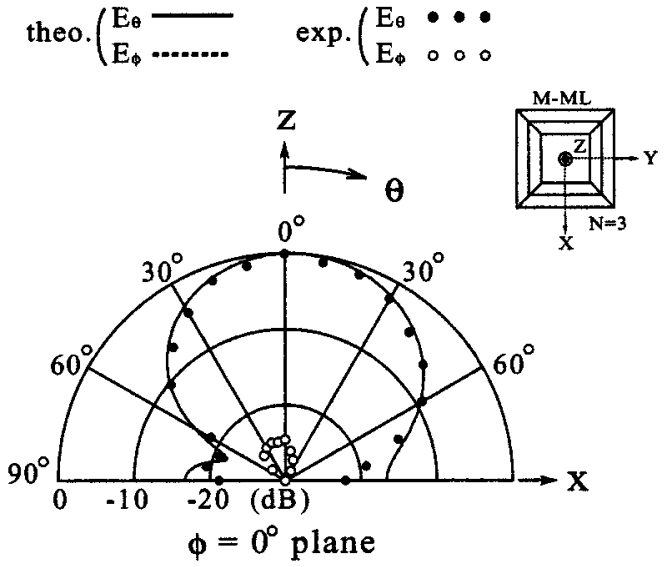

(a)

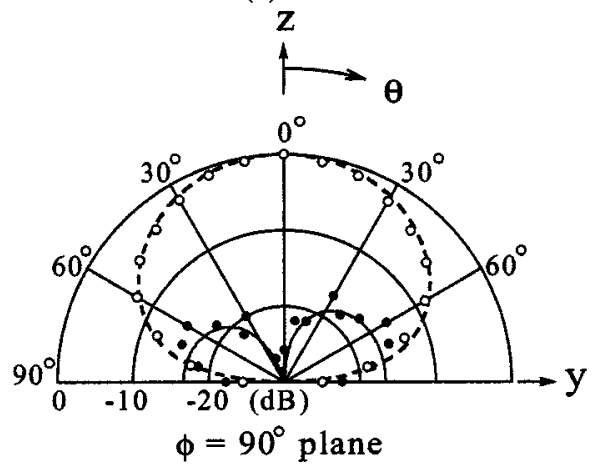

(b)

Fig. 9. Radiation patterns of a modified multiloop antenna with $N=3$ at 3 $\mathrm{GHz}$

$J_{x} \mathbf{x}+J_{y} \mathbf{y}$, with $\mathbf{x}$ and $\mathbf{y}$ being unit vectors) along the loop at $f=3 \mathrm{GHz}$. In this case, the $L$ wire has a total length of approximately one-quarter-wavelength at $3 \mathrm{GHz}\left(L_{V}+L_{H}=\right.$ $\left.0.058 \lambda_{3}+0.197 \lambda_{3}=0.255 \lambda_{3}\right)$. It is found that the current is concentrated along the conductor edges.

Fig. 12(a) shows the theoretical and experimental results of the $\operatorname{VSWR}_{50}$. The frequency bandwidth for a $\operatorname{VSWR}_{50}=2$ criterion is calculated to be $16.7 \%$, which is slightly larger than the value of $15.9 \%$ obtained for a modified ML antenna with $N=7$.

The radiation pattern of the PL is similar to that of the modified ML antenna. Owing to the similarity of the radiation patterns and input impedances of the PL and modified ML antennas, the gain characteristics are also similar. The bandwidth for a 3-dB gain drop criterion is calculated to be approximately $35 \%$ with a maximum gain of approximately $9 \mathrm{~dB}$.

So far, the width of the plate has been fixed to be $W=0.04 \lambda_{3}$ and a VSWR frequency bandwidth of $16.7 \%$ (for a VSWR $=2$ criterion) has been obtained. Note that the VSWR bandwidth increases as $W$ is increased. For example, when the width is increased to $W=0.065 \lambda_{3}$, the VSWR bandwidth increases to $21.5 \%$ with $L_{V}+L_{H}=0.064 \lambda_{3}+0.190 \lambda_{3}=0.254 \lambda_{3}$. Also, note that, as seen from Fig. 12(b), a wide VSWR characteristic similar to that in Fig 12(a) is obtained with a feed wire whose horizontal section is parallel to the $x$-axis [15] (see the inset of Fig. 12(b), where $L_{V}+L_{H}=0.084 \lambda_{3}+0.171 \lambda_{3}=0.255 \lambda_{3}$ ).

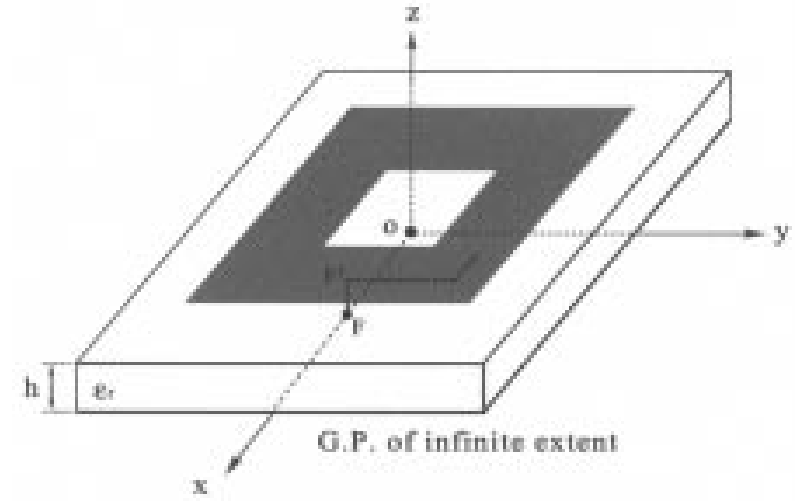

(a)

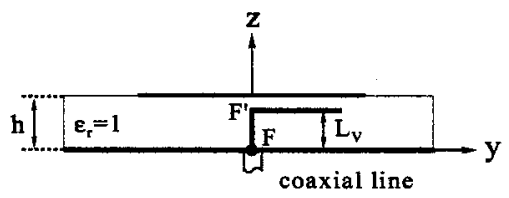

(b)

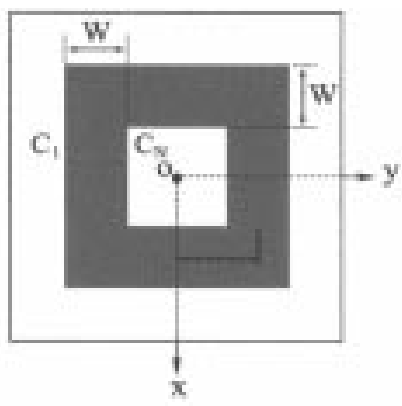

(c)

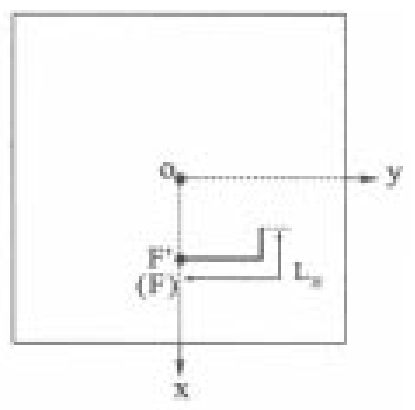

(d)
Fig. 10. Configuration and coordinate system of a plate-loop antenna having a ground plane of infinite extent. (a) Perspective view. (b) Cross-sectional view. (c) Top view of plate loop. (d) Top view of $L$-shaped wire.
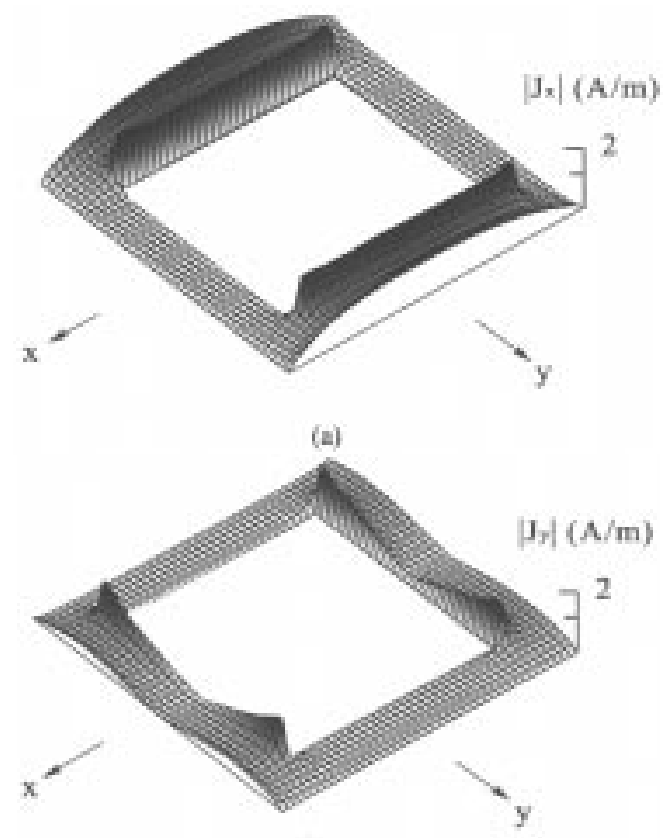

(b)

Fig. 11. Electrical current density along a plate loop; (a) $\left|J_{z^{2}}\right|$, and (b) $\left|J_{y}\right|$. 


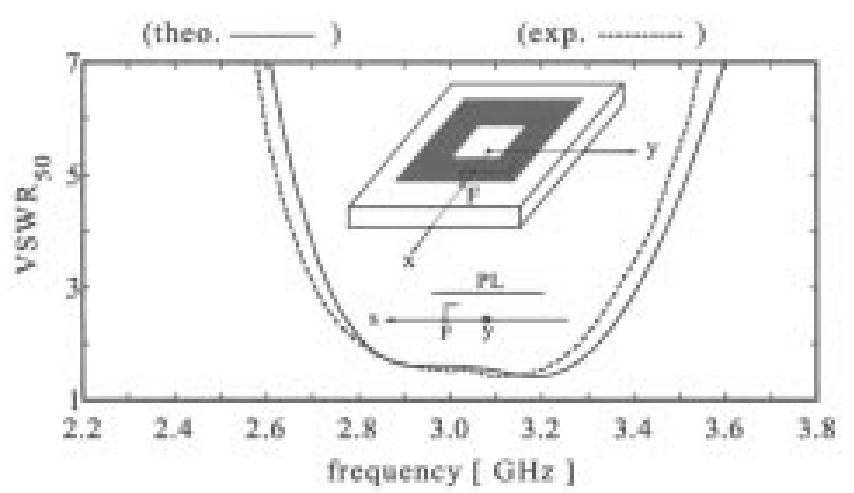

(a)

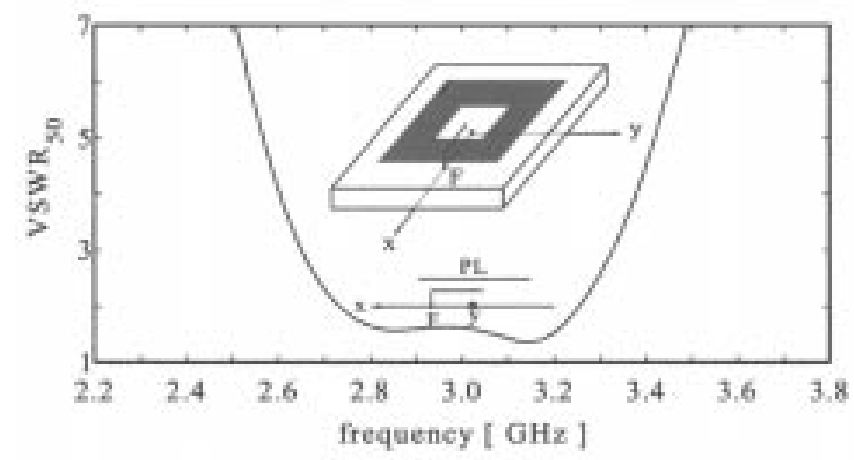

(b)

Fig. 12. VSWR's (relative to a $50 \Omega$ line) of a plate-loop antenna excited with (a) the $L$ wire shown in Fig. 10(d), and (b) a wire whose horizontal section of length $L_{H}$ is parallel to the $x$-axis.

It should, however, be mentioned that the feed wire whose horizontal section is parallel to the $x$-axis cannot realize wide-band VSWR and multifrequency characteristics in the modified ML and discrete ML antennas, respectively.

\section{CONCluSiON}

A discrete ML antenna of $N$ loops has been analyzed. It has been shown that the discrete ML antenna has $N$ minima in the VSWR frequency response curve. A VSWR minimum occurs when the circumference of one of the $N$ loops corresponds to an electrical length of approximately one wavelength. This leads to the fact that the radiation patterns are similar at frequencies giving the VSWR minima. A modified ML antenna has also been analyzed. The modified ML antenna has the same structure as the discrete ML antenna except that the corners of the $N$ loops are connected by wires. The frequency bandwidth for a $\operatorname{VSWR}_{50}=2$ criterion is evaluated to be $14.9 \%$ for $N=3,15.8 \%$ for $N=5$, and $15.9 \%$ for $N=7$. These bandwidths are more than 2.5 times as wide as the bandwidth of the single loop (5.7\% for $N=1$ ).

Further investigation has been directed toward a PL antenna, which corresponds to the structure when $N$ is infinite in the modified ML antenna case. The PL antenna has VSWR and radiation patterns similar to those of the modified ML antennas. Owing to these facts, the difference in the frequency response of the gains for the PL and modified ML antennas is small. The PL has a bandwidth of approximately $35 \%$ for a $3-\mathrm{dB}$ gain drop criterion with a maximum gain of approximately $9 \mathrm{~dB}$.

\section{ACKNOWLEDGMENT}

The authors would like to thank V. Shkawrytko for his kind assistance in the preparation of this manuscript.

\section{REFERENCES}

[1] R. C. Johnson, Antenna Engineering Handbook, 3rd ed, NY: McGrawHill, 1993, ch. 5 .

[2] H. Nakano, "A numerical approach to line antennas printed on dielectric materials," Comp. Phys. Commun., vol. 68, no. 1-3, pp. 441-450, Nov. 1991.

[3] K. Kubota, T. Yanagimichi, and R. Sato, "Characteristics of Concentric Loops With a Single Driven Loop," Institute of Electronics and Communication Engineers, Japan, Tech. Rep., Oct. 1981. A.P81-86.

[4] H. Nakano, H. Yoshida, and Y. Wu, "C $\mathrm{C}$-figured loop antenna," Electron. Lett., vol. 31, no. 9, pp. 693-694, Apr. 1995.

[5] H. Nakano, M. Yamazaki, and J. Yamauchi, "Electromagnetically coupled curl antenna," Electron. Lett., vol. 33, no. 12, pp. 1003-1004, Apr. 1997.

[6] H. Nakano, S. Tajima, K. Nakayama, and J. Yamauchi, "Numerical analysis of honeycomb antennas with an electromagnetic coupling feed system," in Proc. Inst. Elec. Eng.-Microw. Antennas Propagat., vol. 145, Feb. 1998, pp. 99-103.

[7] K. M. Luk, C. L. Mak, Y. L. Chow, and K. F. Lee, "Broadband microstrip patch antenna," Electron. Lett., vol. 34, no. 15, pp. 1442-1443, July 1998

[8] K. M. Luk, Y. X. Guo, K. F. Lee, and Y. L. Chow, " $L$-probe proximity fed $U$-slot patch antenna," Electronics letters, vol. 34, no. 19, pp. 1806-1807, Sept. 1998.

[9] E. Yamashita, Ed., Analysis Methods for Electromagnetic Wave Problems. Norwood, MA: Artech House, 1996, vol. 2, ch. 3. H. Nakano.

[10] R. F. Harrington, Field Computation by Moment Method. New York: Macmillan, 1968.

[11] A. Taflove, Computational Electrodynamics. Norwood, MA: Artech House, 1995.

[12] K. S. Yee, "Numerical solution of initial boundary value problems involving Maxwell's equations in isotropic media," IEEE Trans. Antennas Propagat., vol. AP-14, pp. 302-307, May 1966.

[13] Z. P. Liao, H. L. Wong, B. P. Yang, and Y. F. Yuan, "A transmitting boundary for transient wave analysis," Science Sinica, ser. A, vol. 27, no. 10, pp. 1063-1076, 1984.

[14] C. A. Balanis, Antenna Theory, NY: Harper and Row, 1982, ch. 11.

[15] H Nakano, "Circularly polarized conical beam formation and antennas," Patent appl. (Japan) H01-299272, Nov. 1989.

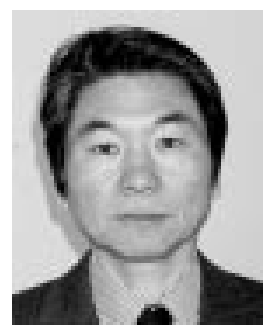

Hisamatsu Nakano (M'75-SM'87-F'92) was born in Ibaraki, Japan. He received the B.E., M.E., and Dr.E. degrees in electrical engineering from Hosei University, Tokyo, in 1968, 1970, and 1974, respectively.

Since 1973, he has been a member of the faculty of Hosei University, where he is now a Professor of Electronic Informatics. His research topics include numerical methods for antennas, electromagnetic wave scattering problems, and light wave problems. He has published more than 170 refereed journal papers and 130 international symposium papers on antenna and relevant problems. He is the author of Helical and Spiral Antennas (New York: Research Studies Press, Wiley, 1987). He also published the chapter "Antenna analysis using integral equations," in Analysis Methods of Electromagnetic Wave Problems, Vol. 2 (Norwood, MA: Artech House, 1996). He was a Visiting Associate Professor at Syracuse University, Syracuse, NY, from March to September 1981, a Visiting Professor at University of Manitoba, Winnipeg, MB, Canada, from March to September 1986, and a Visiting Professor at the University of California, Los Angeles, from September 1986 to March 1987.

Dr. Nakano received an International Scientific Exchange Award from the Natural Sciences and Engineering Research Council of Canada in 1986. In 1987, he received the Best Paper Award from the IEE 5th International Conference on Antennas and Propagation. In 1994, he received the IEEE Antennas and Propagation Society (AP-S) Best Application Paper Award (H.A. Wheeler Award). $\mathrm{He}$ is an Associate Editor of IEEE Antennas and Propagation Magazine, and a member of the AP-S Administrative Committee. 


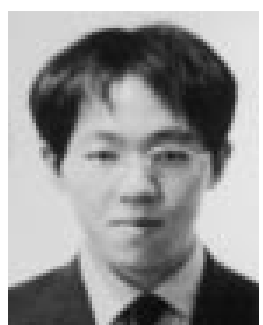

Mitsutoshi Fukasawa received the B.E. and M.E. degrees from Hosei University, Tokyo, Japan, in 1999 and 2001, respectively.

He joined the Oki Co. Ltd., Tokyo, Japan, in 2001. $\mathrm{He}$ is a member of the Institute of Electronics, Information, and Communication Engineers (IEICE) of Japan.

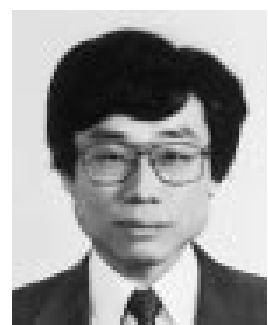

Junji Yamauchi (M'85) was born in Nagoya, Japan, in 1953. He received the B.E., M.E., and Dr.E. degrees from Hosei University, Tokyo, Japan, in 1976, 1978, and 1982, respectively.

From 1984 to 1988 , he served as a Lecturer in the Electrical Engineering Department of Tokyo Metropolitan Technical College, Tokyo, Japan. Since 1988, he has been a member of the faculty of Hosei University, where he is now a Professor of Electronic Informatics. His research interests include optical waveguides and circularly polarized antennas. $\mathrm{He}$ is the author of Propagating Beam Analysis of Optical Waveguides (London, U.K.: Research Studies Press, Wiley, 2002).

Dr. Yamauchi is a member of the Optical Society of America (OSA) and the Institute of Electronics, Information, and Communication Engineers (IEICE) of Japan. 